\title{
CIDI 2017

\section{Visualização de dados sobre os parques públicos cariocas Data Visualization on public parks in Rio de Janeiro}

\author{
Victória D. Sacagami, Larissa E. Silveira, Lucia M. Costa, Julie A. Pires
}

visualização de dados, parques públicos, urbanismo, paisagismo, rio de janeiro

\begin{abstract}
Este artigo tem como objetivo apresentar o desenvolvimento de uma visualização de dados acerca de uma pesquisa sobre a distribuição espacial dos parques públicos da cidade do Rio de Janeiro. Trata-se de um trabalho interdisciplinar, na interface entre Comunicação Visual, Urbanismo e Paisagismo. A partir da visualização de dados, o trabalho visa a criação de uma solução gráfica capaz de revelar espacialidades, inter-relações e dinâmicas socioambientais, levantando discussões acerca dos principais aspectos que influenciam na localização dos parques na cidade.
\end{abstract}

data visualization, public parks, urbanism, landscaping, Rio de Janeiro

This paper aims to present the development of a data visualization about a research on the spatial distribution of public parks in the city of Rio de Janeiro. It is an interdisciplinary work, in the interface between Visual Communication, Urbanism and Landscape Architecture. Based on data visualization, the work aims to create a graphic solution able of revealing spatialities, social relationships and dynamics, raising discussions about the main aspects that influence park location within the city.

\section{Introdução}

A visualização de dados tem se tornado um meio significativo para a disseminação da informação de uma forma eficiente, em um mundo onde ocorre a produção massiva de dados a cada dia. Seu caráter interdisciplinar permite trabalhar os mais variados assuntos, de forma a transformar uma gama de informações em algo visivelmente eficaz, seja para um público leigo ou não. Sendo assim, a visualização de dados surge como uma solução capaz de suprir a necessidade de exibir dados distintos entre si, e permitir uma análise detalhada e comparativa entre os mesmos. Sua aplicação na presente pesquisa fez-se necessária para revelar e esclarecer as possíveis correlações entre os parques e a população em seu entorno, além de localizá-los na cidade do Rio de Janeiro.

A cidade do Rio de Janeiro conta com mais de 30 parques públicos distribuídos pelo seu tecido urbano. Sua importância tem sido largamente apontada por pesquisas voltadas para o estudo das áreas verdes públicas nas cidades. São equipamentos implantados e mantidos pelo poder público, e acessíveis a todos os habitantes da cidade. Mas será que todos têm igual acesso aos parques? Pesquisas também têm demonstrado que parques são fundamentalmente equipamentos de proximidade e que, de um modo geral, valorizam o local onde são implantados. Um estudo sobre a distribuição espacial destes parques pode contribuir para o entendimento das relações que eles estabelecem com a cidade e sua população.

A pesquisa, nas áreas de Urbanismo e Paisagismo, estudou a distribuição dos parques urbanos na cidade do Rio de Janeiro a partir de suas correlações espaciais entre parques, número de habitantes e distribuição de renda, vinculando ainda a data de criação e a área dos parques. O trabalho revelou uma desequilibrada distribuição dos parques, que estão concentrados nas áreas de maior renda e menor população.

Os resultados do estudo sobre a distribuição espacial dos parques foi apresentado inicialmente em dois mapas que relacionavam os parques pelo seu tamanho, data de criação, 


\section{CIDI2017 $\quad 8^{\text {th }} \mathrm{CIDI}$ NATAL

localização na Região Administrativa; um com a renda da população e outro com número de habitantes. O resultado deste mapeamento foi bastante insatisfatório, e assim buscou-se uma nova maneira de representar os mesmos dados, a partir de uma nova cartografia e visualização de dados.

Figura 1: Exemplo de um dos mapas antigos.

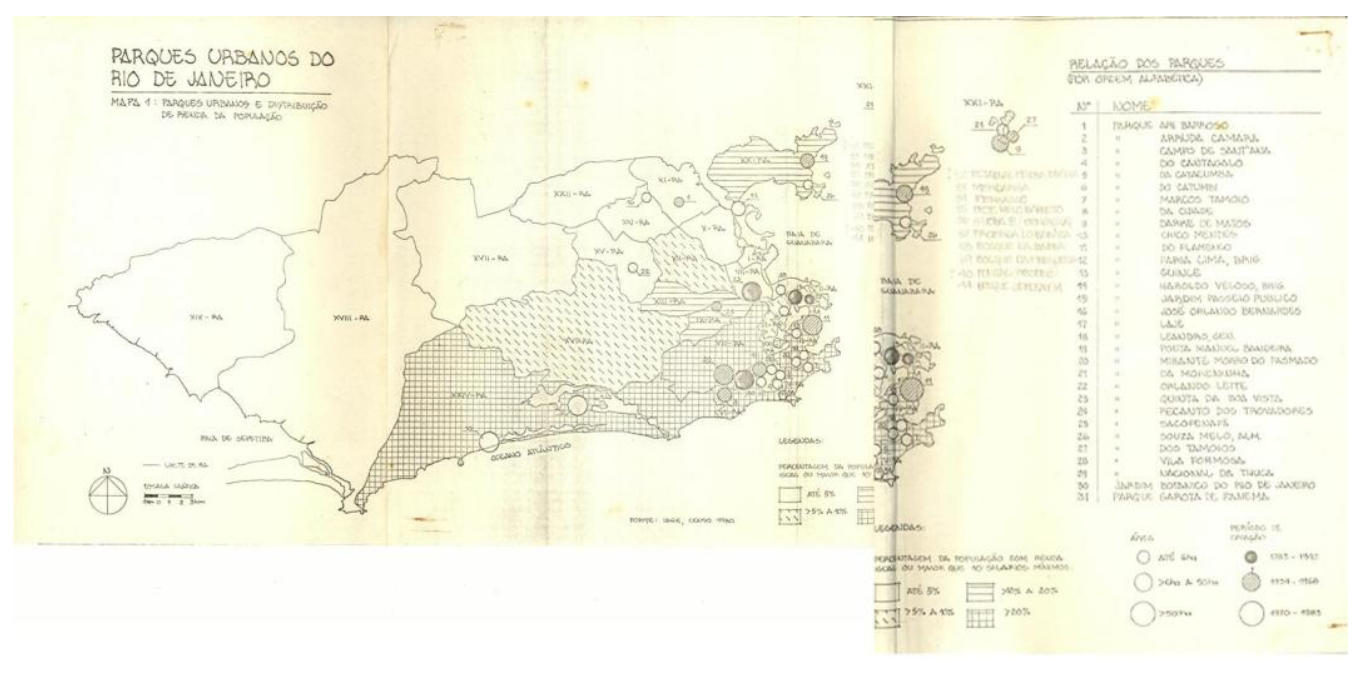

\section{Metodologia}

Inicialmente, foi necessário atualizar os dados da pesquisa já realizada, e incluir os parques naturais da cidade do Rio de Janeiro, além dos parques urbanos. Ambos são destinados ao uso público, porém os parques naturais possuem um caráter ecológico e de pesquisa, enquanto os parques urbanos são destinados prioritariamente para o lazer da população. Foram relacionados 24 parques urbanos e 23 parques naturais, formando um universo de 57 parques a serem mapeados no total.

Para a nova cartografia foi mantido o mesmo critério do mapeamento anterior, ou seja, a divisão do município pelas suas Áreas de Planejamento. O Rio de Janeiro é dividido em cinco Áreas de Planejamento (A.P.'s), subdivididas em 33 Regiões Administrativas (R.A.'s), onde por sua vez encontram-se os 160 bairros cariocas. Essa divisão administrativa é feita de acordo com a proximidade e as suas particularidades urbanas, assim podendo atender as necessidades da cidade de forma geral. A primeira parte do trabalho teve como foco a representação, no mapa, das A.P.'s e suas respectivas regiões administrativas. As A.P.'s foram classificadas por cores, sendo estas aplicadas tanto para a representação dos parques de acordo com suas A.P.'s, quanto para a região em si. 


\section{CIDI 2017

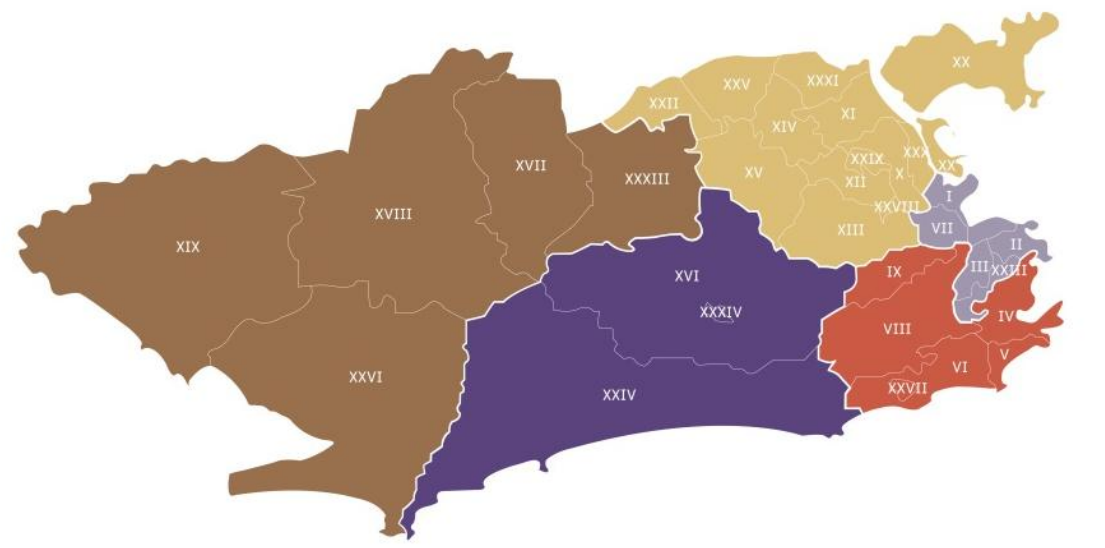

A coleta de dados foi realizada a partir do entendimento da diferença entre Parques Urbanos e Parques Naturais. Foram criadas duas listas distintas, onde reuniu-se informações referentes a cada parque e a região administrativa (RA) onde se localizam.

A gestão desses parques ocorre por diferentes órgãos do governo, sendo os principais a Secretaria Municipal do Meio Ambiente (SMAC), responsável pelos parques naturais, e a Fundação Parques e Jardins (FPJ), administrando os parques urbanos. A coleta de dados deuse então através de revisões bibliográficas, iconográficas, plataformas digitais disponibilizadas pela prefeitura do Rio, SMAC , FPJ, IBGE, consulta em outros órgão públicos e também pelo contato com funcionários da própria prefeitura.

Assim, as informações foram reunidas de acordo com sua relevância para o trabalho, a fim de facilitar a divisão em subgrupos, permitindo uma melhor compreensão de como lidar e relacionar os dados mais importantes, para posteriormente desenvolver uma visualização. Foram coletados:

- Área do parque: Compreender a dimensão de cada parque e sua possível importância a nível ambiental e social.

- Ano de criação: Entender a dinâmica do desenvolvimento da cidade, a fim de descobrir se há alguma relação com a criação dos parques.

- Localização (A.P./R.A./Bairro): Teve como fim identificar a distribuição espacial de cada parque e se existe relação com o entorno (correlaciona-se com Renda/População).

- Renda per capita/população (dados baseados nas R.A.'s): compreender se existe alguma relação com a localização dos parques, que são destinados à população, e se de fato sua distribuição é feita de acordo com o número de habitantes da R.A., ou de acordo com a renda per capita do local. 


\section{CIDI 2017

A partir das análises, foram levantados questionamentos acerca dos fatores que influenciam diretamente na localização dos parques, e se eles estão realmente distribuídos de acordo com as necessidades da população. Houve a confirmação de que determinadas áreas do Rio de Janeiro, são sim privilegiadas de acordo com a renda per capita, sendo elas alvos de maior investimento da prefeitura. Ressaltando novamente o fato de que regiões com menor renda, e consequentemente maior população, acabam sendo desprivilegiadas

Figura 3: Primeira visualização com todas as informações coletadas.

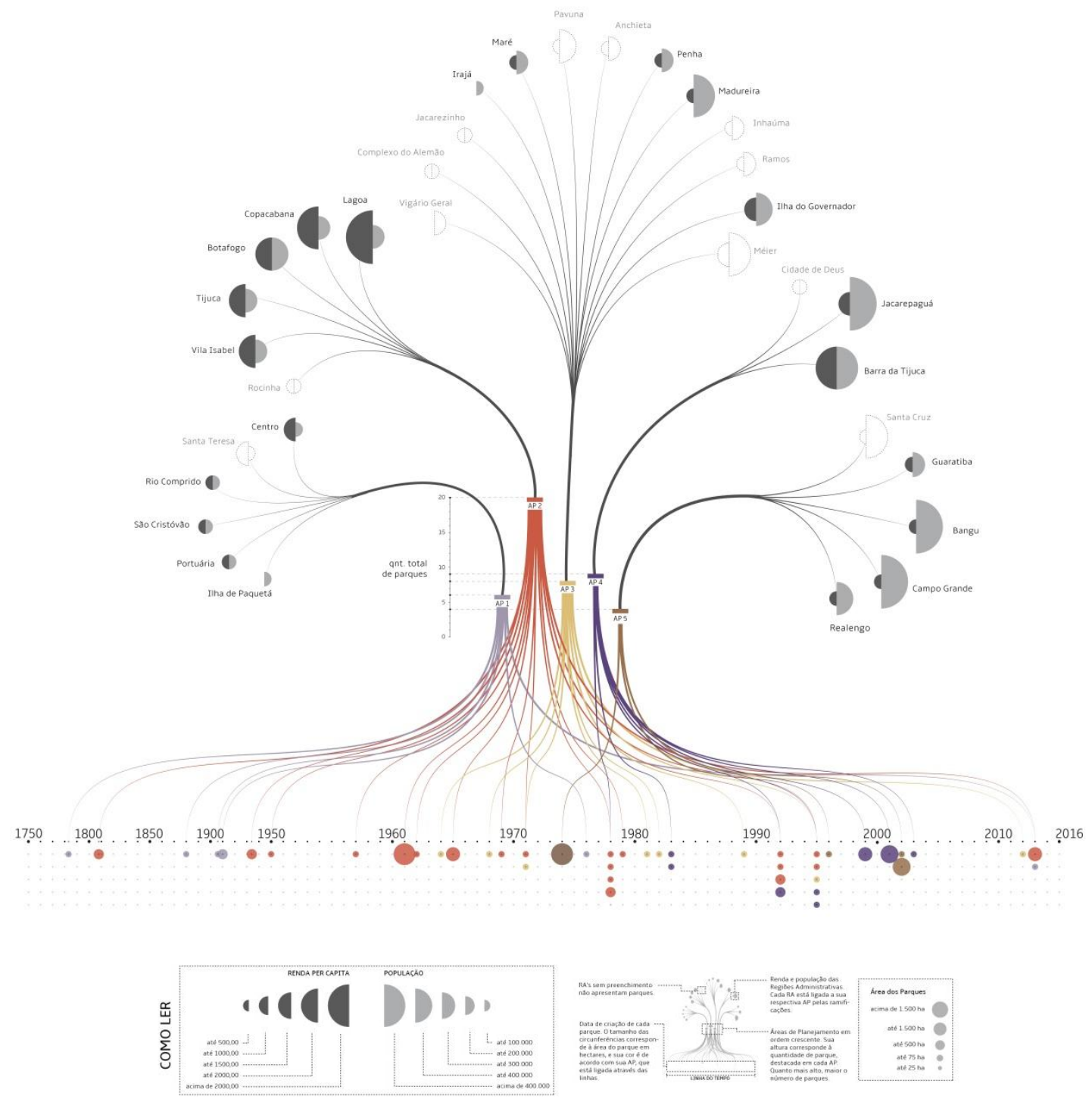




\section{CIDI $20178^{8 \text { " } 1 \text { CIII }}$

No mapa abaixo referente a população, quanto mais escuro maior a quantidade de pessoas na R.A., e no referente a renda per capita, quanto mais escuro, maior a renda da região.

Figura 4: Cartografia com a distribuição espacial dos parques.

Parques Urbanos e Naturais

População
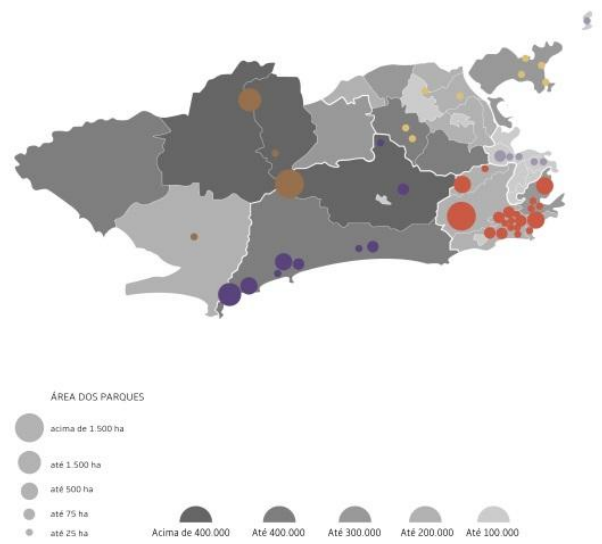

Renda per Capita

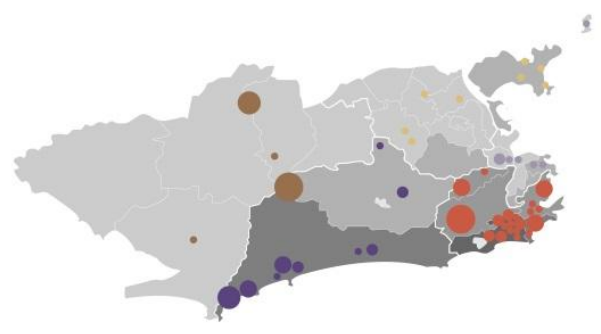

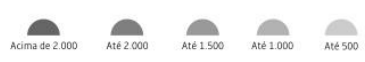

Figura 5: Ao clicar nas R.A.'s, a imagem é direcionada para a localização da região no mapa.
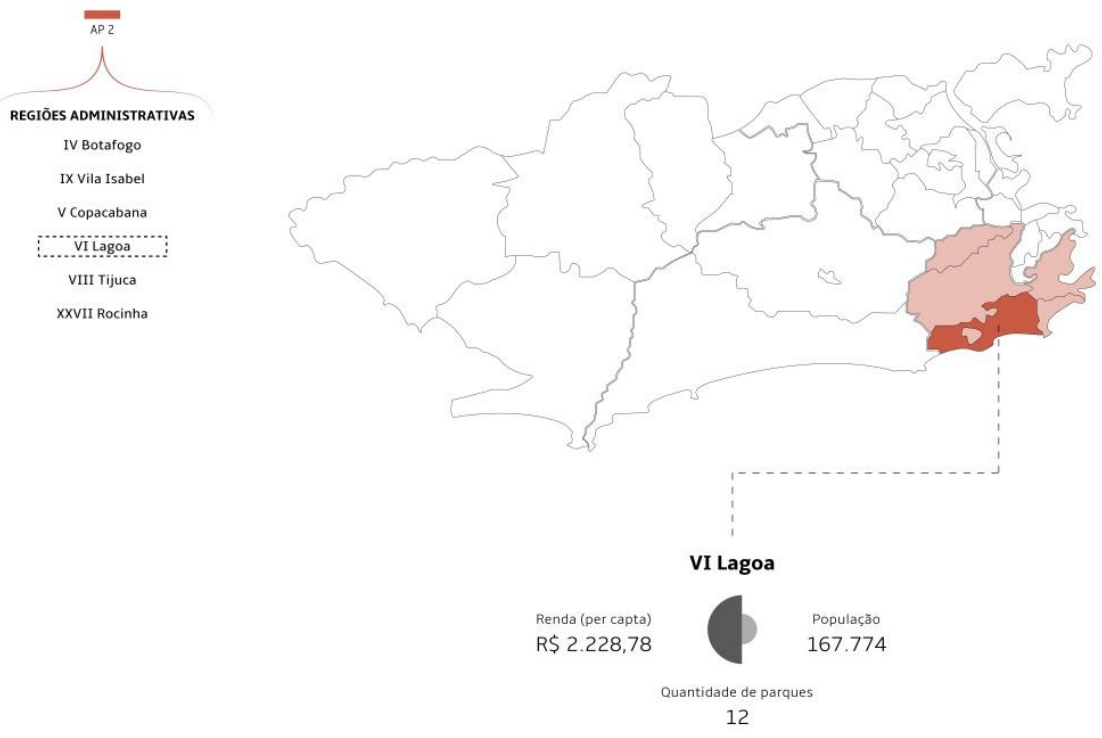


\section{CIDI 2017

Figura 6: Ao clicar na A.P., localizada na parte central da visualização, é possível analisar cada uma individualmente.

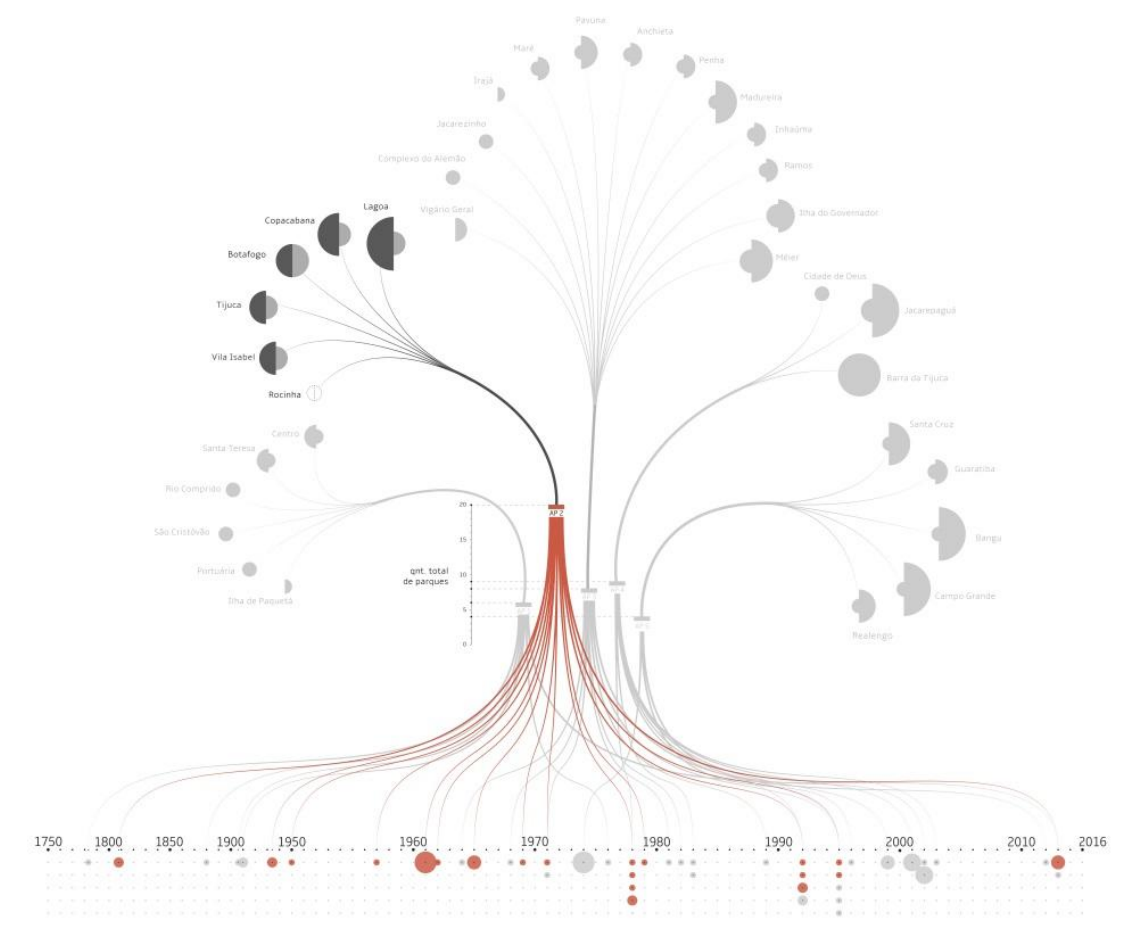

Figura 7: Ao clicar nos parques, localizados na linha do tempo, é direcionado para sua localização na cartografia, junto com informações mais detalhadas sobre ele e parques na sua mesma A.P.

\section{Considerações finais}

Considerando o objetivo de melhor apresentar os resultados de uma pesquisa no âmbito de Urbanismo e Paisagismo, a interdisciplinaridade da visualização de dados com outras áreas de 


\section{CIDI 2017

conhecimento, e a sua importância para a representação de diferentes tipos de informação, foram essenciais para o entendimento e análise desse projeto.

É importante ressaltar também, o papel do designer na sociedade. O design atrelado a outras esferas de pesquisa, se torna um componente significativo para o desenvolvimento e auxílio na transmissão mais eficiente da mensagem, facilitando a compreensão da população acerca de temas mais complexos. Então, com a união desses dois campos nesse trabalho, os resultados do estudo sobre a distribuição espacial dos parques na cidade do Rio de Janeiro ficaram mais claros, legíveis e portanto mais acessíveis tanto ao público leigo quanto aos técnicos do poder público. Assim, estas informações poderão ser melhor utilizadas para orientar políticas públicas que favoreçam a implantação de novos parques na cidade, considerando suas principais carências e necessidades, além de corrigir distorções históricas.

\section{Agradecimento}

As autoras agradecem ao CNPq, FAPERJ e UFRJ pelo apoio à esta pesquisa.

\section{Referências}

COSTA, Lucia M. S. A. Popular values and meanings for urban parks: a case study of Parque do Flamengo in Rio de Janeiro. PhD Thesis, University College London. 1993.

COSTA, Lucia M. S. A. Parques públicos contemporâneos no Rio de Janeiro: a contribuição de Fernando Chacel. In Schicchi, M.C. e Benfatti, D. (org) Urbanismo: Dossiê São Paulo- Rio de Janeiro. Campinas: PUCCCAMP/PROURB, 2004, pp. 275-286.

JACOBSON, R. \& WURMAN, R. 1999. Information Design. Cambridge, MA: MIT Press.

MANOVICH, L. 2010, What is Visualization? Em: Articles: What is visualization? $<$ http://manovich.net/index.php/projects/what-is-visualization>, 04/05/2017.

TUFTE, Edward. The Visual Display of Quantitative Information. Cheshire, CT: Graphics Press.

YAU, N. 2013, Data Points: Visualization that means something. Estados Unidos: John Willey \& Sons, Inc.

\section{Sobre os autores}

Victória Sacagami, bolsista de Iniciação Científica (PIBIC), Curso de Comunicação Visual, EBAUFRJ, Brasil, <victoriasacagami@gmail.com>

Larissa Silveira, bolsista de Iniciação Científica (PIBIC), Curso de Comunicação Visual, EBAUFRJ, Brasil, <larissaelisa@ufrj.br>

Lucia Costa, professora Titular de Paisagismo, Programa de Pós-Graduação em Urbanismo, FAU-UFRJ, Brasil, <lucialice@gmail.com>

Julie Pires, PhD, EBA-UFRJ, Brasil, <juile.pires@eba.ufrj.br> 